\title{
Synthetic Approaches to Complex Organic Molecules in the Cold Interstellar Medium
}

\author{
Eric Herbst ${ }^{1,2 *}$ and Robin T. Garrod ${ }^{1,2}$ \\ ${ }^{1}$ Department of Chemistry, University of Virginia, Charlottesville, VA, United States, ${ }^{2}$ Department of Astronomy, University of \\ Virginia, Charlottesville, VA, United States
}

OPEN ACCESS

Edited by:

Cristina Puzzarini,

University of Bologna, Italy

Reviewed by:

Martin Robert Stewart McCoustra, Heriot-Watt University,

United Kingdom

Barbara Michela Giuliano,

Max Planck Institute for Extraterrestrial

Physics, Germany

${ }^{*}$ Correspondence:

Eric Herbst

eh2ef@virginia.edu

Specialty section:

This article was submitted to

Astrochemistry,

a section of the journal

Frontiers in Astronomy and Space

Sciences

Received: 04 October 2021 Accepted: 01 November 2021

Published: 03 January 2022

Citation:

Herbst E and Garrod RT (2022)

Synthetic Approaches to Complex

Organic Molecules in the Cold Interstellar Medium.

Front. Astron. Space Sci. 8:789428. doi: 10.3389/fspas.2021.789428
The observation and synthesis of organic molecules in interstellar space is one of the most exciting and rapidly growing topics in astrochemistry. Spectroscopic observations especially with millimeter and submillimeter waves have resulted in the detection of more than 250 molecules in the interstellar clouds from which stars and planets are ultimately formed. In this review, we focus on the diverse suggestions made to explain the formation of Complex Organic Molecules (COMs) in the low-temperature interstellar medium. The dominant mechanisms at such low temperatures are still a matter of dispute, with both gas-phase and granular processes, occurring on and in ice mantles, thought to play a role. Granular mechanisms include both diffusive and nondiffusive processes. A granular explanation is strengthened by experiments at $10 \mathrm{~K}$ that indicate that the synthesis of large molecules on granular ice mantles under space-like conditions is exceedingly efficient, with and without external radiation. In addition, the bombardment of carbon-containing ice mantles in the laboratory by cosmic rays, which are mainly high-energy protons, can lead to organic species even at low temperatures. For processes on dust grains to be competitive at low temperatures, however, non-thermal desorption mechanisms must be invoked to explain why the organic molecules are detected in the gas phase. Although much remains to be learned, a better understanding of low-temperature organic syntheses in space will add both to our understanding of unusual chemical processes and the role of molecules in stellar evolution.

Keywords: complex organic molecules, interstellar medium, astrochemistry, nondiffusive motion, radiative association, radiolysis

\section{INTRODUCTION}

Although found in a wide variety of interstellar regions, "complex" organic molecules (abbreviated COMs or iCOMs) were first detected in and are still associated with warm, dense concentrations of gas and dust within interstellar clouds, known as hot cores, that are actively forming stars (Herbst and van Dishoeck 2009). The partially saturated aliphatic COMs are certainly not complex by terrestrial standards, but range in size from 6-13 atoms and contain oxygen and nitrogen as well as carbon and hydrogen. Examples include dimethyl ether $\left(\mathrm{CH}_{3} \mathrm{OCH}_{3}\right)$, methyl formate $\left(\mathrm{HCOOCH}_{3}\right)$, and $n$ - and iso-propyl cyanide $\left(\mathrm{C}_{3} \mathrm{H}_{7} \mathrm{CN} ;\left(\mathrm{CH}_{3}\right)_{2} \mathrm{CHCN}\right)$. In general, $\mathrm{COMs}$ tend to be terrestrial-like in nature, whereas another class of molecules, known as "carbon chains" are quite exotic. Carbon chains are linear and unsaturated molecules consisting mainly of carbon atoms, including negative and positive ions as well as radicals such as $\mathrm{C}_{n} \mathrm{H}(\mathrm{n}=2-8)$ and the cyanopolyynes $\mathrm{HC}_{2 \mathrm{n}} \mathrm{CN}(\mathrm{n}=1-5)$. 
Carbon chains were initially associated exclusively with gasphase formation reactions, especially via ion-molecule reactions, in cold cores at $10 \mathrm{~K}$. The ion-molecule synthesis terminates when positive ions react with electrons in dissociative recombination reactions (Herbst and Millar 2008; Herbst 2017). As a simple example regarding hydrocarbons, consider the reaction

$$
\mathrm{C}^{+}+\mathrm{C}_{2} \mathrm{H}_{2} \rightarrow \mathrm{C}_{3} \mathrm{H}^{+}+\mathrm{H}
$$

followed by the dissociative recombination reaction

$$
\mathrm{C}_{3} \mathrm{H}^{+}+\mathrm{e}^{-} \rightarrow \mathrm{C}_{3}+\mathrm{H}
$$

Such processes can lead to increasingly unsaturated species as size grows, unless reactions between ions and $\mathrm{H}_{2}$ can counter them (e.g., $\mathrm{C}_{3} \mathrm{H}^{+}+\mathrm{H}_{2}$ ). These ion $-\mathrm{H}_{2}$ reactions often do not occur, however (McEwan et al., 1999). Nowadays, it is understood that neutral-neutral gas-phase chemistry can also play a significant role in the gas-phase formation of carbon chains.

Unlike the case of carbon chains, the formation of COMs has been thought to occur mainly during the stellar warm up from cold cores to hot cores initially involving diffusive reactions on grains leading to more saturated species, albeit for most species only strongly at higher temperatures than $10 \mathrm{~K}$ (Viti et al., 2004). These reactions proceed mainly through recombination of radical species on granular surfaces; the precursor radicals are formed from more standard species by photodissociation (Garrod, 2008; Garrod et al., 2008; Garrod 2013). As star-formation proceeds and temperatures rise, thermal desorption gradually occurs, until by $100 \mathrm{~K}$, COMs can be detected in the gas phase.

Although for some time the gradual warm up of star-forming cores was thought to be a pre-requisite for COM chemistry, it was eventually realized that the warm-up scenario was not the whole story because a number of gaseous COMs-principally dimethyl ether and methyl formate-were detected in the gas of cold cores at temperatures such as $10 \mathrm{~K}$, which are lower than believed efficient for COM production on grains (Bacmann et al., 2012). Suggestions were then made that gas-phase processes could lead to these COMs at such low temperatures (Balucani et al., 2015). The major process suggested is known as radiative association, and is discussed below. Other suggestions involving novel surface processes have also been made (Jin and Garrod 2020), and the situation remains unresolved today. Meanwhile, carbon-chain species were also found in warmer regions than cold cores, and this chemistry, named warm carbon-chain chemistry, is thought to start with the desorption of volatile species on icy dust grains such as methane at temperatures as low as $30 \mathrm{~K}$, which initiates a second phase of carbon chain formation via gas-phase ionneutral processes (Sakai and Yamamoto 2013).

Most recently, following infrared detection of a general class of polycyclic aromatic hydrocarbons (PAH's) without the ability to observe individual PAH's, a third class of molecules involving small gaseous aromatic species, e.g., benzonitrile $\left(\mathrm{C}_{6} \mathrm{H}_{5} \mathrm{CN}\right)$ and cyano-naphthalene $\left(\mathrm{C}_{10} \mathrm{H}_{7} \mathrm{CN}\right)$, was detected via rotational emission, first in the cold core TMC-1 (McGuire et al., 2018; McGuire et al., 2021) and subsequently in other sources as well (Burkhardt et al., 2021). The syntheses of these species, typically containing a $\mathrm{CN}$ group to increase their polarity, are not well understood. Indeed, instead of the standard bottom-up chemistry, it has even been suggested that a top-down chemistry is responsible. Other possibilities are based on the reactions of benzene with carbon chains (Shingledecker et al., 2021).

In this minireview, we focus on the production of COMs in low temperature regions. The order of presentation of the diverse synthetic mechanisms thought to produce COMs at low temperatures is as follows: 1) new mechanisms involving nondiffusive processes to produce COMs on cold grains, 2) radiolysis caused by cosmic ray bombardment on icy grains, and 3) gas phase processes, mainly radiative association, a type of reaction in which two molecules collide to form a complex, which can be stabilized by emission of radiation, normally by vibrational emission in the ground electronic state (Herbst 1980a; Herbst, 1980b; Herbst 1985; Vuitton et al., 2012).

\section{FORMATION ON COLD GRAIN SURFACES}

It has been assumed for quite some time that surface reactions proceed via a random diffusive motion in which atoms or molecules move from site to site over barriers known as diffusive barriers, which are often estimated at $0.3-0.5$ of the desorption energy. At very low temperatures, only weakly bound atoms and diatomic species, with low diffusive barriers, can move rapidly enough to be a major cause of reaction. Nevertheless, there is good experimental evidence that moving $\mathrm{H}$ atoms can hydrogenate atoms or diatoms into saturated species such as the conversion of $\mathrm{CO}$ into methanol and of $\mathrm{C}$ atoms into methane (Watanabe and Kouchi 2002). The newly produced molecules can be ejected from the surface of the icy mantle by reactive desorption if the reactions are sufficiently exothermic. Other desorption mechanisms include photodesorption, and cosmic ray desorption (radiolysis), which can occur via sputtering or heating of the whole grain. These diffusive reactions involving $\mathrm{H}$ atoms and heavier species are probably not enough to produce COMs larger than methanol at temperatures of $10 \mathrm{~K}$, although some suggestions to this effect have been made (Chuang et al., 2017). More evidence indicates that the lowest temperatures at which diffusion is important with the exception of hydrogen are $20 \mathrm{~K}$ and higher. For example, diffusive neutral-neutral surface reactions involving radicals are thought to be dominant from 20 to $30 \mathrm{~K}$ upwards.

A recent suggestion based on some initial research (Fedoseev et al., 2015, 2017; Theulé et al., 2013; see also; Theulé 2020) concerns surface reactants that can undergo reactions without significant motion if they lie close enough together. This idea has been worked out in some detail and further refined by Jin and Garrod (2020). One mechanism includes an initial diffusive reaction involving a light atom such as hydrogen to form a product, which upon formation lies adjacent to another atom or molecule, which can lie beneath the newly formed product or next to it. The adjacent species are close enough to react without undergoing any diffusion, and so the second reaction happens "instantaneously" unless there is some chemical activation energy. For example, the surface formation of 


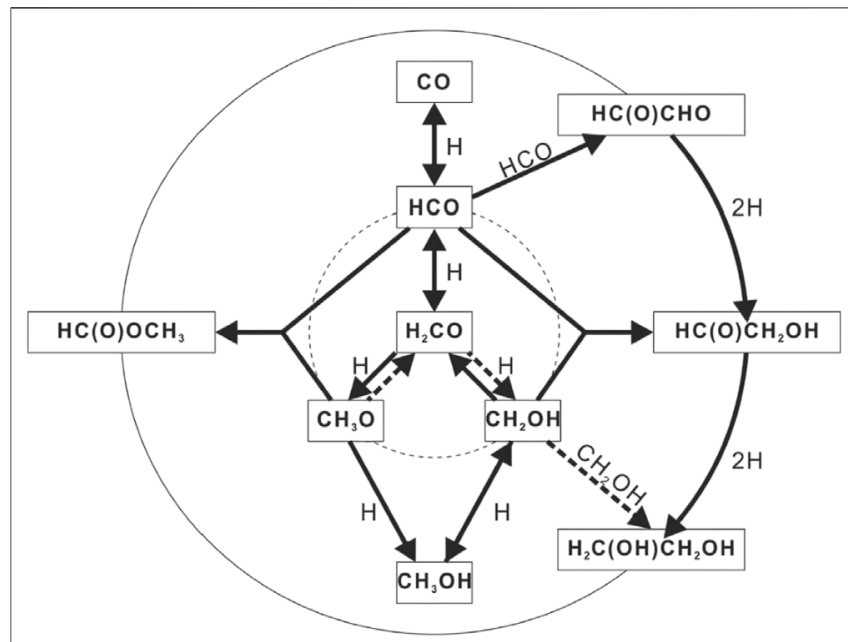

FIGURE 1 | Surface synthesis of glycolaldehyde, ethylene glycol, and possibly methyl formate at low temperatures, as studied by the Leiden group. Figure copied by permission of Oxford University Press from the article by Chuang et al. in Monthly Notices of the Royal Astronomical Society, vol. 455, page 702 (2015).

$\mathrm{CO}_{2}$ from $\mathrm{CO}+\mathrm{O}$ has both diffusive barriers and a chemical activation barrier so is quite slow. But if we consider the diffusive process between atomic oxygen and hydrogen to produce the radical $\mathrm{OH}$, which is rapid, and a subsequent nondiffusive "instantaneous" reaction between the newly formed $\mathrm{OH}$ with an adjacent $\mathrm{CO}$, a reaction leading to $\mathrm{CO}_{2}+\mathrm{H}$, the process is certainly enhanced in rate. This series of two processes is known as a three-body process, in analogy to such a process in the gas. Another "three-body" mechanism consists of an initial photodissociation to cause a stable surface molecule to dissociate into a radical, which is formed next to or over another species, so that a near instantaneous reaction can take place. It is currently unclear if, as mentioned by reviewer 1, water ice has a catalytic effect on nondiffusive motion, similar to the local effect of liquid water. As for the utility of TST, which the reviewer also mentions, we use a variant for diffusive motion leading to reaction, which is a different process.

A third process starts with a metastable species, also formed by photodissociation. A prime example is the metastable $\mathrm{O}\left({ }^{1} \mathrm{D}\right)$ atom, formed directly on the surface through photodissociation of $\mathrm{O}_{2}$, which lives long enough (approx. 1,000 s) to react with virtually any species next to it or lying right below it. The reaction between photodissociatively-formed $\mathrm{O}\left({ }^{1} \mathrm{D}\right)$ and methane has been studied in the laboratory at low temperatures by Bergner et al. (2017); the reaction leads to surface methanol and formaldehyde. Reactions involving $\mathrm{O} \quad\left({ }^{1} \mathrm{D}\right)$ and larger hydrocarbons are likely to eventually lead to COMs. Carder et al. (2021) studied the analogous surface process involving methane that would occur in the cold interstellar medium and found that the mechanism leading to methanol formation is virtually equal in rate to the standard synthesis of the hydrogenation of surface CO via multiple diffusive hydrogen atom reactions albeit at somewhat higher temperatures $(20 \mathrm{~K})$.
Some of the "three-body" surface mechanisms can be much faster than normal diffusion at low temperatures, although are not always so, and so are now regarded as a likely source of oxygen-containing COMs in cold sources as well as sources undergoing warm up. A number of experiments, including those with and without radiation (Chuang et al., 2016, 2017; Fedoseev et al., 2015, 2017), have shown that during deposition on cold icy surfaces, reactions on surfaces can lead to COMs rather quickly; an initial process is shown in Figure 1. In order for the process shown to be efficient, the mechanism is likely to occur via rapid nondiffusive processes, although the details of the processes, which lead to species as large as glycine, are not fully understood (Ioppolo et al., 2021).

\section{RADIOLYSIS}

Cosmic rays are high energy nuclei and electrons with relativistic velocities at nearly the speed of light, with a wide range of energies ranging from $\mathrm{keV}$ to much higher values. The most abundant constituents of the cosmic ray spectrum are simple protons, and their strong interaction with granular ice mantles, an example of radiolysis, can produce COMs from carboncontaining species via sequences of reactions involving the intermediate formation of electrons, ions, and radicals, as the cosmic rays penetrate through the ice. Many experiments have been done, mainly with either high energy protons or electrons, but the details of the chemistry have been mainly lacking until quite recently. The dominant parameter obtained in experiments is known as the $\mathrm{G}$ factor, which is the number of molecules produced or destroyed per $100 \mathrm{eV}$ of incoming energy. Matching experimental and theoretical G values is a necessity for understanding the complex chemistry. The successful sequence of reactions introduced by Shingledecker and Herbst (2018) is as follows. Starting with a species in or on an ice mantle, which is labeled $\mathrm{A}, \mathrm{MeV}$ protons produce $\mathrm{A}^{+}$ions and electrons; these can recombine to form highly electronically excited neutrals $A^{\star}$, which can partially dissociate into $\mathrm{B}^{\star}$ and $\mathrm{C}^{\star}$ excited neutral radicals. Less energetic fragments, designated $B$ and $C$, can be produced by reactions between weakly excited A species and electrons to form excited species $A^{\star}$, which, however, can dissociate into weakly excited $\mathrm{B}$ and $\mathrm{C}$. The excited species $A^{\star}, B^{\star}$, and $C^{\star}$, known as suprathermal (electronically excited) neutral species, can react with thermal neutral species without barriers because the suprathermal energy exceeds any activation energy, thus forming the final radiolysis products. Theoretical radiolysis papers published concerning the formation of COMs include Shingledecker et al., 2018 and the recent paper by Paulive et al. (2021), which shows that radiolysis greatly enhances the rate of production of methyl formate, glycolaldehyde, and acetic acid at low temperatures. Wakelam et al. (2021) have recently published a paper on cosmic ray sputtering emphasizing the importance of experimental work. 


\section{LOW-TEMPERATURE GAS-PHASE PROCESSES}

Radiative association can occur between ions and neutral species and between pairs of neutral species. Both of these processes can lead to hydrogen-rich products if the reactants themselves are well saturated; the products can either be COMs or precursors to COMs. But, in the case that the product is an ion, dissociative recombination with electrons can reduce the saturation significantly since the products tend to fragment more significantly than the loss of a single hydrogen atom from the molecular skeleton.

To determine whether radiative association is a useful approach to low temperature COM production in space, it is necessary to have some knowledge of the reaction rate coefficients. It is, however, very difficult to measure radiative association in the laboratory because it can be detected only at very low densities and because it occurs rather slowly unless the following conditions are met: very low density, very low temperature, a deep potential well, strong infrared emission, and a complex with a large enough number of atoms to last for a relatively long time before redissociation, so that radiative stabilization can occur. Complications include competitive product channels, sometimes accompanied by roaming. Nevertheless, with a very low density experimental apparatus such as found in an ion trap, it is possible to measure the association rate for ion-neutral systems in the radiative, or low density, regime, or to extrapolate from the border of the ternary regime (Gerlich and Hornung 1992). At higher densities, radiative association is indeed eclipsed by ternary association, in which the complex is stabilized by collisions. This stabilization can occur at densities as low as $10^{10}-10^{11} \mathrm{~cm}^{-3}$. At even higher densities ternary association is saturated when all collisions lead to the product. In the density range between radiative association and ternary association (up to circa $10^{16} \mathrm{~cm}^{-3}$ ) it is possible to convert a measured ternary association reaction into radiative association by an assortment of approximate methods (Smith and Adams 1981; Gerlich and Hornung 1992).

In addition there are several purely theoretical approaches to obtain low temperature radiative association rate coefficients, one of which, a phase space approach, calculates the rate of production directly using a long-range capture potential (Herbst 1985) and the other, an RRKM or a variable transition state approach, which calculates the rate of complex dissociation followed by detailed balancing to obtain the radiative association rate (Ryzhov et al., 1996; Vuitton et al., 2012).

It may be possible to form reasonable abundances of COMs via radiative association between the methyl ion $\left(\mathrm{CH}_{3}^{+}\right)$and larger neutrals despite the subsequent destruction of the ion product via dissociative recombination with electrons. The fact that the methyl ion can be depleted by reaction with $\mathrm{H}_{2}$ albeit slowly makes it less abundant by 1-2 orders of magnitude than the $\mathrm{C}^{+}$ion throughout much of the lifetime of a cold core. Nevertheless, this abundance can lead to the moderate production of some COMs.

The simplest radiative association involving methyl ions occurs with $\mathrm{H}_{2}$ :

$$
\mathrm{CH}_{3}^{+}+\mathrm{H}_{2} \rightarrow \mathrm{CH}_{5}^{+}+h v
$$

This system has been studied by ternary conversion and by theory (Gerlich and Hornung 1992). Once produced, $\mathrm{CH}_{5}{ }^{+}$can undergo dissociative recombination to produce the dominant heavy products $\mathrm{CH}_{3}$ and $\mathrm{CH}_{2} ; \mathrm{CH}_{4}$ is detected at only approximately $5 \%$ of the total carbonaceous products (Semaniak et al., 1998). This low branching fraction is likely to be common. A quite recent approach to low-temperature COM production can bypass the dissociative recombination step if there is a sufficient abundance of ammonia, based on the fact that ammonia has a very high proton affinity (Taquet et al., 2016.) Reactions between protonated molecules and ammonia will form the neutral molecule exclusively (along with $\mathrm{NH}_{4}^{+}$), unlike the case of dissociative recombination. Consider the formation of dimethyl ether from the precursor protonated ion via dissociative recombination:

$$
\mathrm{CH}_{3} \mathrm{O}(\mathrm{H}) \mathrm{CH}_{3}^{+}+\mathrm{e}^{-} \rightarrow \mathrm{CH}_{3} \mathrm{OCH}_{3}+\mathrm{H} \text {, }
$$

where the measured branching fraction for the dimethyl ether channel is a low 0.07 (Hamberg et al., 2010). On the other hand, the competitive proton exchange reaction with ammonia

$$
\mathrm{CH}_{3} \mathrm{O}(\mathrm{H}) \mathrm{CH}_{3}^{+}+\mathrm{NH}_{3} \rightarrow \mathrm{CH}_{3} \mathrm{OCH}_{3}+\mathrm{NH}_{4}^{+}
$$

is exothermic by $50 \mathrm{~kJ} / \mathrm{mol}$, and produces dimethyl ether. Depending on the abundance of ammonia, reaction (5) and similar reactions with other protonated species can affect the production of COMs (Taquet et al., 2016; Garrod et al., 2021). Ammonia abundances may be too low, however, other than in hot cores or shocked regions.

Many other examples of COMs produced via the $\mathrm{CH}_{3}{ }^{+}$ radiative association-dissociative recombination sequence have been discussed in the literature, some based on experiments involving ternary systems or low-density experiments such as achieved in ion-cyclotron resonance machines, some obtained by theory, and some simply estimated. Examples include methanol, ethanol, acetonitrile, propionitrile, and methyl isocyanide. Smith and Adams, 1981, Gerlich and Hornung (1992), Smith (1992), and Huntress and Mitchell (1979) have reported and tabulated a significant number of radiative association reactions between $\mathrm{CH}_{3}{ }^{+}$and large neutral species leading to COMs. Dissociative recombination measurements involving storage rings exist but are not common, necessitating estimates of branching fractions. The reliability of some of the radiative association research is uncertain. Despite this problem, radiative association experiments involving ions are infrequently undertaken today, yet a renewal of interest in ion-molecule radiative association reactions would probably be needed to revive interest in the importance of the subject in the interstellar medium.

The case of neutral-neutral radiative association reactions had not been considered significantly in an astronomical context until quite recently (Balucani et al., 2015). No experimental measurements exist in this field to the best of our knowledge, but theoretical calculations are being undertaken. One example concerns the radiative association between the radicals $\mathrm{CH}_{3}$ and $\mathrm{CH}_{3} \mathrm{O}$ to produce dimethyl ether: 


$$
\mathrm{CH}_{3}+\mathrm{CH}_{3} \mathrm{O} \rightarrow \mathrm{CH}_{3} \mathrm{OCH}_{3}+h v,
$$

The methoxy radical can be produced by the reaction between $\mathrm{OH}$ and $\mathrm{CH}_{3} \mathrm{OH}$, which has been studied at a variety of temperatures by several groups using the CRESU apparatus. The reaction is quite rapid at $11.7 \mathrm{~K}$ in the laboratory so can occur at the $10 \mathrm{~K}$ temperature in cold cores (Ocaña et al., 2019). As regards the production of dimethyl ether, the phase space theoretical approach of Herbst (1985) has been utilized, and shows a rate coefficient for radiative association of only slightly below the collisional limit $-3 \times 10^{-11} \mathrm{~cm}^{3} \mathrm{~s}^{-1}$ - at a temperature of $10 \mathrm{~K}$. Once dimethyl ether is formed, it can lead to the production of methyl formate by reacting with halogen atoms to form the radical $\mathrm{CH}_{3} \mathrm{OCH}_{2}$, which then reacts with atomic oxygen to form methyl formate $\left(\mathrm{HCOOCH}_{3}\right)$ (Balucani et al., 2015). When put into current simulations of cold core chemistry, this sequence comes somewhat behind granular processes in forming the observed values of these two COMs, which are the two dominant larger COMs found in cold cores. Similar neutralneutral radiative association processes have been invoked to occur in the upper reaches of the molecule-rich atmosphere of Titan, the large moon of Saturn (Vuitton et al., 2012).

To make further progress in extending the use of neutralneutral gas-phase chemistry to form cold COMs, one possibility is to consider radical-radical radiative association reactions analogous to reaction (6). As an example, one could study the reaction between the hydroxymethyl radical $\left(\mathrm{CH}_{2} \mathrm{OH}\right)$ and formyl radical ( $\mathrm{HCO})$ to form glycolaldehyde $\left(\mathrm{CH}_{2} \mathrm{OHCHO}\right)$, or the reaction of the hydroxymethyl radical and methyl to produce ethanol. Analogous association reactions can also occur on granular surfaces, where the excess reaction energy can be transferred to the grains. At $10 \mathrm{~K}$, however, heavy species diffuse only slowly. To enhance the rate of COM formation on

\section{REFERENCES}

Bacmann, A., Taquet, V., Faure, A., Kahane, C., and Ceccarelli, C. (2012). Detection of Complex Organic Molecules in a Prestellar Core: a New challenge for Astrochemical Models. AઐA 541, L12. doi:10.1051/0004-6361/ 201219207

Balucani, N., Ceccarelli, C., and Taquet, V. (2015). Formation of Complex Organic Molecules in Cold Objects: the Role of Gas-phase Reactions. MNRAS 449, L16-L20. doi:10.1093/mnrasl/slv009

Bergner, J. B., Öberg, K. I., and Rajappan, M. (2017). Methanol Formation via Oxygen Insertion Chemistry in Ices. ApJ 845, 29. doi:10.3847/1538-4357/ aa7d09

Burkhardt, A. M., Loomis, R. A., Shingledecker, C. N., Lee, K. L. K., Remijan, A. J., McCarthy, M. C., et al. (2021). Ubiquitous Aromatic Carbon Chemistry at the Earliest Stages of star Formation. Nat. Astron. 5, 181-187. doi:10.1038/s41550020-01253-4

Carder, J. T., Ochs, W., and Herbst, E. (2021). Modelling the Insertion of $\mathrm{O}\left({ }^{1} \mathrm{D}\right)$ into Methane on the Surface of Interstellar Ice Mantles. MNRAS 508, 1526-1532. doi:10.1093/mnras/stab2619

Chuang, K.-J., Fedoseev, G., Ioppolo, S., van Dishoeck, E. F., and Linnartz, H. (2016). H-atom Addition and Abstraction Reactions in Mixed CO, H2CO and $\mathrm{CH} 3 \mathrm{OH}$ Ices - an Extended View on Complex Organic Molecule Formation. MNRAS 455, 1702-1712. doi:10.1093/mnras/stv2288

Chuang, K.-J., Fedoseev, G., Qasim, D., Ioppolo, S., van Dishoeck, E. F., and Linnartz, H. (2017). Production of Complex Organic molecules: H-Atom grain surfaces, one must consider a faster approach to surface chemistry - surface chemistry without diffusion - as discussed above.

\section{DISCUSSION}

In this brief minireview, we have discussed some of the synthetic approaches to the production of COMs in the cold regions of interstellar clouds. At present, it appears as if the granular approaches-nondiffusive processes and irradiation via cosmic ray bombardment-are slightly more efficient than gas-phase reactions, mainly radiative association. But much more research on radiative association reactions is needed to confirm this hypothesis. In the realm of radiative association, it does appear that neutral-neutral systems are more likely to be of more importance than ion-neutral systems, mainly because the products of neutral-neutral reactions do not have to cope with the rapid destruction caused by dissociative recombination.

\section{AUTHOR CONTRIBUTIONS}

EH wrote the initial draft of the manuscript, while RTG revised it and was responsible for the science behind the nondiffusive mechanism.

\section{FUNDING}

This work was funded by the National Science Foundation (US) Division of Astronomical Sciences through Grant AST-19-06489.

Addition versus UV Irradiation. Mon. Not. R. Astron. Soc. 467, 2552-2565. doi:10.1093/mnras/stx222

Fedoseev, G., Chuang, K.-J., Ioppolo, S., Qasim, D., Dishoeck, E. F. v., and Linnartz, H. (2017). Formation of Glycerol through Hydrogenation of CO Ice under Prestellar Core Conditions. ApJ 842, 52. doi:10.3847/1538-4357/aa74dc

Fedoseev, G., Cuppen, H. M., Ioppolo, S., Lamberts, T., and Linnartz, H. (2015). Experimental Evidence for Glycolaldehyde and Ethylene Glycol Formation by Surface Hydrogenation of CO Molecules under Dense Molecular Cloud Conditions. MNRAS 448, 1288-1297. doi:10.1093/mnras/stu2603

Garrod, R. T. (2008). A New Modified-Rate Approach for Gas-Grain Chemical Simulations. AઐA 491, 239-251. doi:10.1051/0004-6361:200810518

Garrod, R. T. (2013). A Three-phase Chemical Model of Hot Cores: The Formation of Glycine. ApJ 765, 60. doi:10.1088/0004-637X/765/1/60

Garrod, R. T., Jin, M., Matis, K. A., Jones, D., Willis, E. R., and Herbst, E. (2021). Formation of Complex Organic Molecules in Hot Molecular Cores through Nondiffusive Grain-Surface and Ice-Mantle Chemistry. arXiv.

Garrod, R. T., Weaver, S. L. W., and Herbst, E. (2008). Complex Chemistry in Starforming Regions: An Expanded Gas-Grain Warm-up Chemical Model. ApJ 682, 283-302. doi:10.1086/588035

Gerlich, D., and Horning, S. (1992). Experimental Investigation of Radiative Association Processes as Related to Interstellar Chemistry. Chem. Rev. 92, 1509-1539. doi:10.1021/cr00015a003

Hamberg, M., Zhaunerchyk, V., Vigren, E., Kaminska, M., Kashperka, I., Zhang, M., et al. (2010). Experimental Studies of the Dissociative Recombination of ions $\mathrm{CD}_{3} \mathrm{OCD}_{2}{ }^{+}$and $\left(\mathrm{CD}_{3}\right)_{2} \mathrm{OD}^{+}$Unknown Node Mtable Found in MathML Fragment. A\&A 522, A90. doi:10.1051/0004-6361/201014774 
Herbst, E. (1980a). A New Look at Radiative Association in Dense Interstellar Clouds. ApJ 237, 462-470. doi:10.1086/157889

Herbst, E. (1980b). An Additional Uncertainty in Calculated Radiative Association Rates of Molecular Formation at Low Temperatures. ApJ 241, 197-199. doi:10.1086/158331

Herbst, E., and Millar, T. J. (2008). "The Chemistry of Cold Interstellar Cloud Cores," in Low Temperatures and Cold Molecules. Editor I. W. M. Smith (London, UK: Imperial College Press), 1-54. doi:10.1142/9781848162105_0001

Herbst, E. (1985). Radiative Association Rate Coefficients under Shocked Conditions in Interstellar Clouds - the Case of $\mathrm{CH}_{3}^{(+)}+\mathrm{H}_{2}$. Astron. Astrophys. 153, 151-156.

Herbst, E. (2017). The Synthesis of Large Interstellar Molecules. Int. Rev. Phys. Chem. 36, 287-331. doi:10.1080/0144235X.2017.1293974

Herbst, E., and van Dishoeck, E. F. (2009). Complex Organic Interstellar Molecules. Annu. Rev. Astron. Astrophys. 47, 427-480. doi:10.1146/annurev-astro-082708101654

Huntress, W. T. J., Jr., and Mitchell, G. F. (1979). The Synthesis of Complex Molecules in Interstellar Clouds. ApJ 231, 456-467. doi:10.1086/157207

Ioppolo, S., Fedoseev, G., Chuang, K.-J., Cuppen, H. M., Clements, A. R., Jin, M., et al. (2021). A Non-Energetic Mechanism for Glycine Formation in the Interstellar Medium. Nat. Astron. 5, 197-205. doi:10.1038/s41550-02001249-0

Jin, M., and Garrod, R. T. (2020). Formation of Complex Organic Molecules in Cold Interstellar Environments through Nondiffusive Grain-Surface and Ice-Mantle Chemistry. ApJS 249, 2630. doi:10.3847/1538-4365/ ab9ec8

McEwan, M. J., Scott, G. B. I., Adams, N. G., Babcock, L. M., Terzieva, R., and Herbst, E. (1999). New $\mathrm{H}$ and $\mathrm{H}_{2}$ Reactions with Small Hydrocarbon Ions and Their Roles in Benzene Synthesis in Dense Interstellar Clouds. ApJ 513, 287-293. doi:10.1086/306861

McGuire, B. A., Burkhardt, A. M., Kalenskii, S., Shingledecker, C. N., Remijan, A. J., Herbst, E., et al. (2018). Detection of the Aromatic Molecule Benzonitrile $\left(\mathrm{c}-\mathrm{C}_{6} \mathrm{H}_{5} \mathrm{CN}\right)$ in the Interstellar Medium. Science 359, 202-205. doi:10.1126/science.aao4890

McGuire, B. A., Loomis, R. A., Burkhardt, A. M., Lee, K. L. K., Shingledecker, C. N., Charnley, S. B., et al. (2021). Detection of Two Interstellar Polycyclic Aromatic Hydrocarbons via Spectral Matched Filtering. Science 371, 1265-1269. doi:10.1126/science.abb7535

Ocaña, A. J., Blázquez, S., Potapov, A., Ballesteros, B., Canosa, A., Antiñolo, M., et al. (2019). Gas-phase Reactivity of $\mathrm{CH} 3 \mathrm{OH}$ toward $\mathrm{OH}$ at Interstellar Temperatures (11.7-177.5 K): Experimental and Theoretical Study. Phys. Chem. Chem. Phys. 21, 6942-6957. doi:10.1039/c9cp00439d

Paulive, A., Shingledecker, C. N., and Herbst, E. (2020). The Role of Radiolysis in the Modelling of $\mathrm{C} 2 \mathrm{H} 4 \mathrm{O} 2$ Isomers and Dimethyl Ether in Cold Dark Clouds. MNRAS 500, 3414-3424. doi:10.1093/mnras/staa3458

Ryzhov, V., Klippenstein, S. J., and Dunbar, R. C. (1996). Radiative Association of $\mathrm{NO}^{+}$with 3-Pentanone: Rate, Binding Energy, and Temperature Dependence. J. Am. Chem. Soc. 118, 5462-5468. doi:10.1021/ja953183b

Sakai, N., and Yamamoto, S. (2013). Warm Carbon-Chain Chemistry. Chem. Rev. 113, 8981-9015. doi:10.1021/cr4001308

Semaniak, J., Larson, A., Le Padellec, A., Strömholm, C., Larsson, M., Rosén, S., et al. (1998). Dissociative Recombination and Excitation of $\mathrm{CH}_{5}{ }^{+}$: Absolute Cross Sections and Branching Fractions. ApJ 498, 886-895. doi:10.1086/ 305581
Shingledecker, C. N., and Herbst, E. (2018). A General Method for the Inclusion of Radiation Chemistry in Astrochemical Models. Phys. Chem. Chem. Phys. 20, 5359-5367. doi:10.1039/C7CP05901A

Shingledecker, C. N., Lee, K. L. K., Wandishin, J. T., Balucani, N., Burkhardt, A. M., Charnley, S. B., et al. (2021). Detection of Interstellar $\mathrm{H}_{2} \mathrm{CCCHC}_{3} \mathrm{~N}$. AઐA 652, L12. doi:10.1051/0004-6361/202140698

Shingledecker, C. N., Tennis, J., Gal, R. L., and Herbst, E. (2018). On Cosmic-Raydriven Grain Chemistry in Cold Core Models. ApJ 861, 2015. doi:10.3847/15384357/aac5ee

Smith, D., and Adams, N. G. (1981). Some Positive Ion Reactions with H2: Interstellar Implications. Monthly Notices R. Astronomical Soc. 197, 377-384. doi:10.1093/mnras/197.2.377

Smith, D. (1992). The Ion Chemistry of Interstellar Clouds. Chem. Rev. 92, 1473-1485. doi:10.1021/cr00015a001

Taquet, V., Wirström, E. S., and Charnley, S. B. (2016). Formation and Recondensation of Complex Organic Molecules during Protostellar Luminosity Outbursts. ApJ 821 (46), 46. doi:10.3847/0004-637X/821/1/46

Theulé, P. (2020). "Chemical Dynamics in Interstellar Ice," in Laboratory Astrophysics: from Observations to Interpretation, IAU Symposium 350. Editors F. Salama and H. Linnartz. doi:10.1017/S1743921319008342

Theulé, P., Duvernay, F., Danger, G., Borget, F., Bossa, J. B., Vinogradoff, V., et al. (2013). Thermal Reactions in Interstellar Ice: A Step towards Molecular Complexity in the Interstellar Medium. Adv. Space Res. 52, 1567-1579. doi:10.1016/j.asr.2013.06.034

Viti, S., Collings, M. P., Dever, J. W., McCoustra, M. R. S., and Williams, D. A. (2004). Evaporation of Ices Near Massive Stars: Models Based on Laboratory Temperature Programmed Desorption Data. Monthly Notices R. Astronomical Soc. 354, 1141-1145. doi:10.1111/j.1365-2966.2004.08273.x

Vuitton, V., Yelle, R. V., Lavvas, P., and Klippenstein, S. J. (2012). Rapid Association Reactions at Low Pressure: Impact on the Formation of Hydrocarbons on Titan. ApJ 744, 11. doi:10.1088/0004-637X/744/1/11

Wakelam, V., Dartois, E., Chabot, M., Spezzano, S., Navarro,-Almaida, D., Loison, J.-C., et al. (2021). Efficiency of Non-thermal Desorptions in Cold-Core Conditions. A\&A 652, A63. doi:10.1051/0004-6361/202039855

Watanabe, N., and Kouchi, A. (2002). Efficient Formation of Formaldehyde and Methanol by the Addition of Hydrogen Atoms to $\mathrm{CO}$ in $\mathrm{H}_{2} \mathrm{O}-\mathrm{CO}$ Ice at $10 \mathrm{~K}$. Astrophys. J. 571, L173-L176. doi:10.1086/341412

Conflict of Interest: The authors declare that the research was conducted in the absence of any commercial or financial relationships that could be construed as a potential conflict of interest.

Publisher's Note: All claims expressed in this article are solely those of the authors and do not necessarily represent those of their affiliated organizations or those of the publisher, the editors, and the reviewers. Any product that may be evaluated in this article or claim that may be made by its manufacturer, is not guaranteed or endorsed by the publisher.

Copyright (c) 2022 Herbst and Garrod. This is an open-access article distributed under the terms of the Creative Commons Attribution License (CC BY). The use, distribution or reproduction in other forums is permitted, provided the original author(s) and the copyright owner(s) are credited and that the original publication in this journal is cited, in accordance with accepted academic practice. No use, distribution or reproduction is permitted which does not comply with these terms. 